\title{
Study on the fatty acid nutrition of colostrum combined with biomedicine.
}

\author{
Aiju Chen', Weili Zhang 2,3,", Jiamin $\mathrm{He}^{3}$, Minghua Jiang ${ }^{3}$ \\ ${ }^{1}$ Obstetrics and Gynecology Hospital of Fudan University, Shanghai 200090, PR China \\ ${ }^{2}$ Xinhua Hospital Affiliated to Shanghai Jiaotong University, School of Medicine, Shanghai 200092, PR China \\ ${ }^{3}$ Shanghai Institute for Pediatric Research, Shanghai 200092, PR China
}

\begin{abstract}
Maternal colostrum is the primary post natal nutrient supply that affects the growth and development of newborns. The study of the fatty acid nutrition of colostrum based on biomedicine is of great significance. In this paper, the problem of fatty acid nutrition of colostrum in combination with biomedicine was analyzed. The study analyses the main contents of biomedicine as well as the variation of the content is analyzed through questionnaire method and big data analysis. The results showed that the nutritional level of colostrum fatty acid is related to the type and quantity of food consumed by pregnant women during pregnancy. And the level of fatty acids is related to the total amount of fat and protein ingested by the mother.
\end{abstract}

Keywords: Biomedicine, Newborn, Fatty acid.

Accepted on May 25, 2017

\section{Introduction}

Maternal colostrum is the most nutritious food for newborns. It serves as the main energy source for the development of infants. Colostrum contains fatty acids which provide the newborn with the necessary energy for healthy growth. The distribution of fatty acids in maternal colostrum is affected by various factors, including maternal, lactation, geographical and dietary habits, etc. [1]. The infant development is mainly depended on the dietary lipids present in the colostrum [2].

Biomedical research in China is still a relatively new research subject. Biomedical research combines medical information, medical imaging, nanotechnology, and materials technology. With the continuous development of information technology and network intelligence, the big data technology has been gradually introduced in biomedical technology. These technologies have played an important role in medical research and diagnosis, which not only improves the accuracy of medical diagnosis, but also greatly reduces the workload of medical data analysis [3].

Therefore, based on the domestic and foreign relevant theories, through the questionnaire survey and the method of big data analysis, the fatty acid nutrition of neonatal colostrum based on biomedicine is analyzed and studied. In the second section, the main contents of biomedicine are expounded. In the third section, the main contents of colostrum are introduced. And the method of large data analysis is used to describe the fatty acid of neonatal colostrum. In section fourth, the data obtained from the analysis of large data is listed and analyzed. Finally, in the fifth section, the conclusions are drawn.

\section{State of the Art}

The research of biomedicine in foreign countries is earlier, while the research of biomedicine in China is relatively late. In recent years, China's biomedical research institutions and enterprises have continued to introduce and learn from foreign technology and equipment, so as to continuously enhance the academic level of China's biomedical field. In the era of intelligent information network, the big data analysis technology has been introduced into the field of biomedical research. This technology has played a decisive role in the research of biomedicine in China, and has optimized the research methods of biomedicine in a large extent. For decades, molecular biology experiments were used to draw conclusions, or to put forward a new hypothesis. And now based on large amounts of biomedical data, the law can be explored, and the assumption or draw reliable conclusions can be put forward.

Colostrum mainly refers to the milk produced by the mother in the first few days before production and continues during the early days of breast feeding. After the delivery, the hormone level is affected by production, then which will begin to secrete milk. The physiological changes of the mother's body facilitate production of colostrum milk which is more suitable for the needs of the newborn. The mode of nutrient delivery is in concentrated to low volume form which makes it more compatible for newborns digestive system [4-6]. The fatty acids in the maternal colostrum are very important for the healthy growth of newborns, mainly because the fatty acids in colostrum can provide the necessary nutrition for the newborn. 


\section{Methodology}

In the era of great material life, people attach great importance to the nutrition of pregnant women and the growth of newborns. Most of the residents in our country provide the most abundant nutrition to the pregnant women. To a certain extent, it has promoted the continuous study of the colostrum in the field of biomedicine. This is mainly because colostrum is recognized as the world's best neonatal food. Colostrum has a short duration, and so the quality of colostrum has a direct effect on the development of the newborn. Colostrum is also rich in immune factors and growth factors, and these nutrients are essential for the healthy growth of newborns [7]. If the newborn can receive a full amount of colostrum fatty acids, then the newborn can have a strong immunity. However, the safety of colostrum depends mainly on the current situation of newborn diet.

$S S=\sum_{i} \frac{\left(\sum_{i} X_{i j}\right)^{2}}{n_{i}}-C \rightarrow(1)$

Based on the research results of biomedicine and the method of large data analysis, the nutritional status of neonatal colostrum fatty acids is analyzed and studied. In this study a used questionnaire survey is used to track and record the number and quantity of food for pregnant women in three different regions. Then, the formula (1) is used to test and analyze the data, and to summarize the nutritional components of colostrum. Finally, the relationship between the level of fatty acid and the main food consumption is studied, to provide new ideas and directions for the future study of neonatal colostrum. To sum up, the use of big data analysis method to study the nutritional level of neonatal colostrum fatty acids can improve the objectivity and accuracy of the results.

\section{Result Analysis and Discussion}

The results obtained by the method described above are shown in the following tables:

Table 1. Analysis of main food consumption of pregnant women.

\begin{tabular}{llll}
\hline Food species & A & B & C \\
\hline Staple food & 216 & 219 & 187 \\
\hline Meat & 88 & 120 & 78 \\
\hline Eggs & 44 & 47 & 59 \\
\hline Vegetables & 262 & 283 & 302 \\
\hline
\end{tabular}

Table 2. Nutritional status of colostrum.

\begin{tabular}{llll}
\hline Nutrition type & A & B & C \\
\hline Energy & 1647 & 1952 & 1750 \\
\hline Protein & 95 & 129 & 102 \\
\hline Fat & 97 & 112 & 107 \\
\hline
\end{tabular}

\begin{tabular}{llll}
\hline Carbohydrate & 353 & 358 & 329 \\
\hline
\end{tabular}

Tables 1 show the consumption of food in pregnant women and the results shows that the consumption level of staple foods such as vegetables and fruits is highest, while the consumption level of meat and eggs is lower. Table 2 shows the nutritional status of colostrum. Data shows the nutritional status of colostrum in pregnant women in different regions. The energy levels of colostrum are comparatively higher than those of other nutrients, followed by the carbohydrates. The nutritional level of protein and fat is lower. Based on the analysis of the data obtained from the biomedical big data technology, it is concluded that the nutritional level of the fatty acid in the newborn colostrum is related to the type and quantity of the food consumed by the pregnant women during pregnancy. The level of fatty acids is related to the total amount of fat and protein.

\section{Conclusion}

With the continuous improvement of living standards in China, people in our country have paid more and more attention to the growth and development of infants and young children. Colostrum is essential for the healthy growth and development of the infants. Therefore, the study on the fatty acid nutrition of newborn colostrum combined with biomedicine is of great significance. Based on the questionnaire and the method of big data analysis, the fatty acid nutrition of neonatal colostrum based on biomedicine was analyzed and studied. At the same time, the process of analyzing the fatty acid of neonatal colostrum was described. Then, the relationship between the fatty acid and the maternal food consumption was summarized. And the reference basis was provided for improving the level of fatty acid in maternal colostrum. It is believed that the level of fatty acids is associated with the total amount of fat and protein ingested by the mother. In order to provide enough colostrum fatty acids to the newborn, the mother should reasonably absorb foods rich in protein and fat during pregnancy.

\section{References}

1. Fainberg $\mathrm{H} \mathrm{P}$, Almond $\mathrm{K} \mathrm{L}, \mathrm{Li} \mathrm{D}$. Impact of maternal dietary fat supplementation during gestation upon skeletal muscle in neonatal pigs. BMC Physiology 2014; 14: 1-12.

2. Jensen RG. Lipids in human milk. Lipids 1999; 34: 1243-1271.

3. Garcia M, Greco LF, Favoreto MG. Effect of supplementing fat to pregnant nonlactating cows on colostral fatty acid profile and passive immunity of the newborn calf. J Dairy Sci 2014; 97: 392-405.

4. Gao YX, Zhang J, Wang CR, Li LX, Man QQ, Song PK, Meng LP, Lie Ø, Frøyland L. The fatty acid composition of colostrum in three geographic regions of China. Asia Pacific J Clin Nutrition 2013; 22: 276-282.

5. Jiang J, Wu K, Yu Z, Ren Y, Zhao Y, Jiang Y, Xu X, Li W, Jin Y, Yuan J, Li D. Changes in fatty acid composition of 
human milk over lactation stages and relationship with dietary intake in Chinese women. Food Function 2016; 7: 3154-3162.

6. Li SY, Dong XL, Wong WS, Su YX, Wong MS. LongChain Polyunsaturated Fatty Acid Concentrations in Breast Milk from Chinese Mothers: Comparison with Other Regions. Int J Child Health Nutrition 2015; 4: 230-239.

7. Hurley WL, Theil PK. Perspectives on Immunoglobulins in Colostrum and Milk. Nutrients 2011; 3: 442-474.

\section{*Correspondence to}

Weili Zhang

Xinhua Hospital Affiliated to Shanghai Jiaotong University School of Medicine

PR China 